\title{
Asymmetry of magnetization curves of textured BSCCO
}

D.M. Gokhfeld, D.A. Balaev, S.I. Popkov, K.A. Shaykhutdinov, M.I. Petrov

L.V. Kirensky Institute of Physics SD RAS, Krasnoyarsk, Russia

\begin{abstract}
The dependence of magnetization on magnetic field $M(H)$ was measured for textured BSCCO samples for $H\|c, H\| a b$ at different temperatures. Inclusion of the pinning parameter in the Valkov-Khrustalev model [V.V. Valkov, B.P. Khrustalev, JETP 80 (1995) 680] allowed to describe successfully the asymmetric $M(H)$ dependencies. The temperature and magnetic field dependencies of critical current and pinning parameter for $H \| c$ and $H \| a b$ were estimated.
\end{abstract}

Key words: magnetization, Bean model, Bi2223, asymmetry, anisotropy, texture

\section{Introduction}

Connection between the field dependence of critical current density $j_{c}(H)$ and the $M(H)$ dependence of superconductors is established in many works. However computation of $M(H)$ curves at different temperatures remains a nontrivial task. The Valkov-Khrustalev model (VK) [1] was applied earlier for description of the $M(H)$ loops of $\mathrm{Tl}$ and Bi based ceramics [1, 2] at low temperatures. At higher temperatures there was serious discrepancies between the theoretical curves and experimental data.

In this paper an anisotropic magnetization of bulk textured $\mathrm{Bi}_{1.8} \mathrm{~Pb}_{0.3} \mathrm{Sr}_{1.9} \mathrm{Ca}_{2} \mathrm{Cu}_{3} \mathrm{O}_{x}$ (BSCCO) ceramics has been studied. Asymmetric magnetization loops were described by the modified version of VK.

\section{Experiment}

Preparation of bulk textured BSCCO samples is described in [3]. In these textured ceramics plate-like BSCCO crystallites have the thickness 1-2 $\mu \mathrm{m}$ along the axis and the average linear dimensions $\sim 10 \mu \mathrm{m} \times 10 \mu \mathrm{m}$ in the 
$a b$ plane. The degree of texture is 0.97 . The critical temperature $T_{c}$ is $113 \mathrm{~K}$ [3].
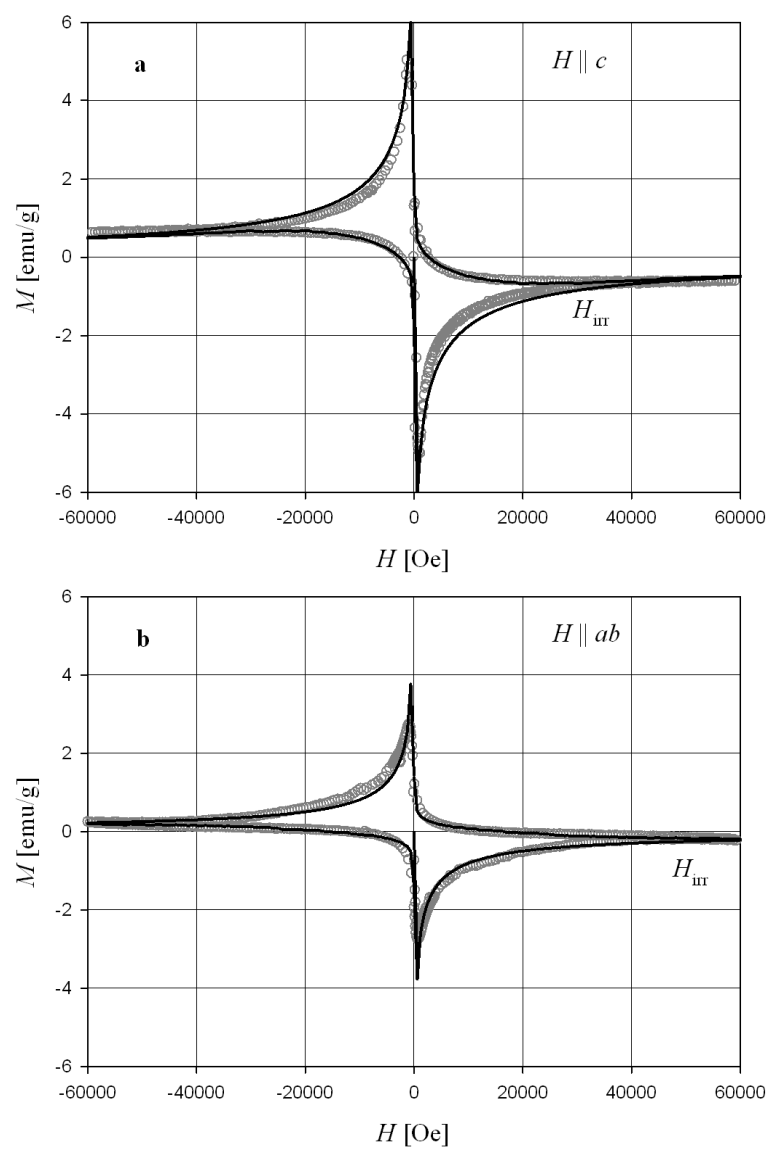

Figure 1: Magnetization vs. magnetic field dependencies for $H \| c$ and $H \| a b(\mathbf{b})$ at $T=40 \mathrm{~K}$. Experiment (points) and computed curves (solid lines).

Magnetic measurements have been performed using the vibration sample magnetometer at temperature range $4.2-80 \mathrm{~K}$. The cubic sample has the size $0.5 \times 0.5 \times 0.5 \mathrm{~mm}^{3}$.

Figure 1 demonstrates the experimental $M(H)$ dependencies (points) for $H \| c$ (a) and $H \| a b(\mathrm{~b})$ at $T=40 \mathrm{~K}$. The diamagnetic response for $H \| c$ in $\approx 2$ times larger than one for $H \| a b$ at all temperatures. The $M(H)$ loop at $T=4.2 \mathrm{~K}$ has a typical form for the hard superconductors: modules of the sample magnetization are about equal when $H$ is increased and when $H$ 
is decreased, $\left|M^{-}\right| \approx M^{+}$, at fields $0 \leq H \leq H_{m}$, here $H_{m}$ is the maximal reached value of applied field. This symmetry of $M(H)$ loop disappears at higher temperatures. We observed an asymmetry $\left|M^{-}\right|>M^{+}$on the $M(H)$ loops measured at $14 \mathrm{~K}$ and higher. At higher temperatures this asymmetry increases. Also the experimental $M(H)$ dependencies demonstrate that the forward and backward values of magnetization coincide starting with $H=$ $H_{i r r}$. The irreversibility field $H_{i r r}$ decreases exponentially as $T$ increases (for

$T \geq 40 \mathrm{~K}$ ). The value of $H_{i r r}$ for $H \| c$ is smaller in $\approx 2$ times than $H_{i r r}$ for $H \| a b$.

\section{Model}

Two substantial modifications of the Bean model were realized in VK [1]: (i) account of a statistical ensemble of granules, (ii) two-scale $j_{c}(H)$ dependence. Influence of a dispersion of the granules sizes is planned to be considered later. Here we imply the negligible dispersion.

VK considers the strong pinning of vortices so as the sample remembers the maximal reached field $H_{m}$ during the field cycling. The value of $H_{m}$ determines the depth of vortex penetration $R_{m}=F\left(H_{m}\right)$, where $F$ is a nondecreasing function (see details in [1], equations $(5),(8)$ ). There are pinned vortices throughout the entire volume of the granules with the radius $R \leq R_{m}$.

Clean low- $T_{c}$ superconductors, where pinning is absent, may have nonhysteretic $M(H)$ curves. The $M(H)$ loops of high- $T_{c}$ superconductors narrow, down to the non-hysteretic dependence, as $T$ increases. So one should account the decrease of pinning causing this narrowing. Let us introduce the parameter $p$ such that $p=1$ for the strong pinning and $p=0$ then vortices are not pinned in a sample. The case $p=0$ means that there is not a memory about magnetic history. Then the sample magnetization is fully determined by the present value of $H$ instead $H_{m}$, also $R_{m}=F(H)$. Selecting of $p$ between 0 and 1 allows to obtain the $M(H)$ dependence for any pinning strength through the superposition of $R_{m}(p=1)$ and $R_{m}(p=0)$ as follows $R_{m}(p)=p F\left(H_{m}\right)+(1-p) F(H)$. The asymmetry of computed curves is now strong determined by $p$. For $p=1$ the loop is symmetric, a decreasing of $p$ increases the asymmetry. Field dependence of this pinning parameter should be selected for the best description of the $M(H)$ loops. Unfortunately we cannot fit exactly the experimental $M(H)$ loops by the single $p$. Reaching of a successful description of a low field part of the loop accompanies with a 
synchronous discrepancy at fields about $H_{m}$ and vice versa. Computations demonstrate that two first integrals of equation (12) of [1] determine $M^{+}$in low fields and two last members determine $M^{+}$near $H_{m}$. So we use parameter $p_{1}$ for low-field part of $M(H)$ and $p_{2}$ for high-field part of $M(H)$. We selected the field dependencies provided the reversible $M(H)$ at fields higher $H_{\text {irr }}$ such that $p_{1,2}(H)=p_{1,2}^{0} \exp \left(-0.55 H / H_{\text {irr }}\right)$.

We believe that the introduced pinning parameters concern the pinning energy and its field and temperature dependencies. Further investigations will be devoted to this question.

\section{Discussion}

The computed curves are displayed in figure 1 a,b with experimental points at $T=40 \mathrm{~K}$. Small observed discrepancies between the curves and experimental data are possible to be result of the form of the sample and the granules. Fitting values of $p_{1}^{0}, p_{2}^{0}$ decrease exponentially as $T$ grows. The $j_{c}(T)$ dependence estimated from the fitting decreases slower than $j_{c}(T)$ estimated from the loop width $\Delta M(H=0, T)=M^{+}-M^{-}$.

In spite of the high degree of texture the observed anisotropy parameter $\gamma \approx 2$ is low comparing with $\gamma$ of the single crystal BSCCO. This is probably because the high sensitivity of the high- $T_{c}$ crystals to smallest misalignments between their axes and the field direction. Similar anisotropy of magnetization of the textured BSCCO samples was observed earlier [4].

In summary, the modification of VK approach [1] is suggested to describe the asymmetric experimental $M(H)$ dependences of high- $T_{c}$ ceramics. The good agreement of the computed curves and the experimental loops is achieved for $H \| c$ and $H \| a b$.

We are thankful to V.V. Valkov for discussions. This work is supported by program N5 of RAS, project N7.

\section{References}

[1] V.V. Valkov, B.P. Khrustalev, JETP 80 (1995) 680

[2] D.M. Gokhfeld, et al, Physica C 434 (2006) 135

[3] M.I. Petrov, et al, Tech. Phys. Lett. 33 (2007) 740

[4] P.J. Kung, et al, Physica C 249 (1995) 53 